\title{
Automation of visualization process for organizational and technological design solutions
}

\author{
Sergei Sinenko ${ }^{1}$, Tatiana Poznakhirko ${ }^{1, *}$, and Vyacheslav Obodnikov ${ }^{1}$ \\ ${ }^{1}$ Department of Technologies and Organizations of Construction Operations, Institute of Construction and Architecture, State \\ University of Civil Engineering, Federal State Budget Educational Institution of Higher Education, Moscow, Russian Federation
}

\begin{abstract}
This article studies modern software packages used in drawing construction master plans and their elements. A special emphasis is placed on increasing the level of design works, improving their quality, and expanding the community of technically unskilled users. The article describes approaches to solving the task of visualization of organizational and technological solutions and gives a comparative analysis thereof. It presents a visualization diagram of an organizational and technological solution for the construction of a building. It also highlights the most promising direction in graphic modeling of a construction process for buildings and structures with visualization seen as the most objective solution to address the assigned task.
\end{abstract}

\section{Introduction}

The quality and cost of an engineering, construction or production project depend, to a large extent, on which design technology it uses. In days gone by, all technical documentation used to be developed manually on drawing boards. At present, with personal computers commonly used by designers and process engineers, any project is unthinkable without computer-aided design (CAD) systems.

This country saw the appearance of such systems as a "progressive tool of acceleration of the work of designer bureaus" in the early 1980 s in the aviation industry. Even though the first attempts did not bring the expected results, they gave impetus to $\mathrm{R} \& \mathrm{D}$ work in this direction. While at first the main task of CAD was to develop external surfaces of machines and machine tools and make strength calculations, soon afterwards these systems "learned" to compute various types of diagrams, make architectural and construction drawings, create legends and indicate dimensions, and even produce fullfledged drawings in line with applicable industry standards.

Application of CAD boosts the performance of a designer or a process engineer two- or three-folds, and raises the efficiency of interaction between various subdivisions and the level and quality of design and engineering works. Moreover, CAD provides means for reducing the duration of fitting-out operations, relieving designers from the need to perform non-productive works, reducing the number of employees, expanding the possibilities of designing and manufacturing sophisticated equipment, as well as establishing an integrated and unified design and technology database of a business. A distinguishing feature of such systems is constant improvement - year by year the functionality of these systems and working speeds of designers/engineers keep steadily growing, therefore number of working specialists is being reduced. All these factors, in turn, produce a favorable impact on a business's financial stance.

The remarkable progress of computing engineering and its sweeping development in the last decades has proven an exceptional role of computers, which, on the one hand, act as a support tool for the design process, and, on the other hand, as an intermediary that drastically boosts the efficiency of human intellectual activities.

Finally, it became possible to use the virtual reality technology as a forming environment. The idea of online design came into being as a result of analysis of the use of virtual technologies as a tool of visualization of design solutions on the organization and technologies of construction operations [1]. It is based on complete immersion of a construction engineer in the environment being designed. The engineer finds himself inside the designed space, determines the direction of changes, and implements all these changes interactively by moving forms in a virtual space. The implementation of the "online design" idea is supported by a large number of virtual reality research. An example of such "immersion" is shown on Figure 1.

\footnotetext{
$\overline{{ }^{*} \text { Corresponding author: top1977@ya.ru }}$
} 


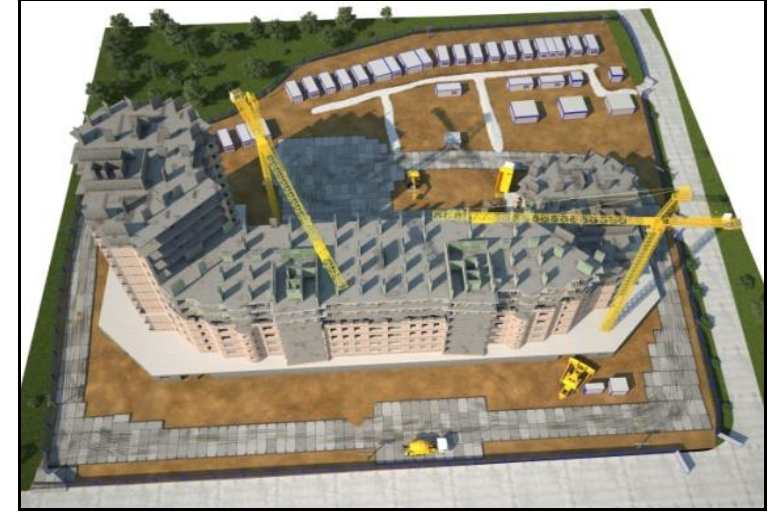

Fig. 1. Master plan of a construction project

\section{Methods, findings and discussion}

Organizational and technological documentation of a Construction Organization Design and a Works Performance Design is known to include a Construction Master Plan, or a site layout plan that depicts the location of permanent buildings and structures, temporary mobile buildings and structures, permanent and temporary railways and automobile roads, utilities, warehouses, building cranes and mechanized units, production base facilities, as well as existing buildings and those subject to demolition.

Up-to-date software packages "Gektor-Constructor" (automated workstation "Works Performance Design", automated workstation "Construction Organization Design", etc.), NanoCAD, "Constructor" and others ensure improved development of construction master plans.

The main rationalization principles in the development of organizational and technological documentation are maximum reduction of manual work of designers, improving labor efficiency in design organizations, decreasing the duration of design operations and raising the quality of design documentation. Improvement tools to be employed in this process include software and hardware packages based on the use of computers, peripheral devices, reprographics and office automation equipment that allow for combining a technological process and document reproduction into a single system [3].

The main prerequisite for rationalization is the use of surface modeling and document templates, electrostatic devices for reproducing originals, microfilms of enlarged copies of documents, as well as aperture cards as a tool ensuring automation of document copying and storing processes.

Software package "Gektor: Construction Designer" offers up-to-date development technologies for all sections of Construction Organization Designs and Works Performance Designs, including construction master plans.

One of the current trends in computer graphics development is photorealistic computer-modelled imaging [1].

This is vital for computer-aided designing, the motion picture industry, advertising, commercial design, computer games, etc. An improved quality of computer graphics products is an increasingly common requirement in practical operations, and the user demand for realistic 3D images is continually growing [2].

Expansion of computing capacities offers solutions to progressively challenging tasks of 3D scene visualization (number of objects, sources of illumination, image resolution); however, it is outpaced by increasing complexity of these tasks.

There are two main approaches to 3D model visualization:

- Generation of pseudo 3D samples to be subsequently processed according to $2 \mathrm{D}$ graphics principles, with limited use of the simplest 3D laws;

- Generation of a 3D world model to be subsequently projected on a flat screen. This is how all modern $3 \mathrm{D}$ games are produced.

\subsection{Animation}

Animation is a complex process of frame-by-frame arrangement of objects of a construction master plan and adjusting the path of their motion and interaction with the other elements of the 3D model, a process that requires attention to particulars. Depending on the computer's capacity, the animation and visualization quality can vary from photorealistic to a schematic display of capabilities of the software package.

Consideration should be given to what is expected at the end of the animation process. It means that the duration of a completed animated product must necessarily be calculated. Preference should be given to advance computations before the animation goes to rendering, because the latter is an extremely long process.

It is a common practice, for computer time and workstation resources saving purposes, to use video editors and to edit completed video files by stretching frames thus slowing down the motion of objects. This approach has both strong and weak points. Its strong point is saving time and resources; its weak point is the resulting video. The required number of frames is not calculated in advance, and the editor has nothing to fill in the gaps between the frames. The outcome is a jagged and jumpy video. It is perfectly clear that a smooth and nice video is much more pleasing both for an external viewer and the interested party.

In view of all the above, it can be concluded that generation of any animation should start with computation of time and the number of frames. Thus, for a five-minute animation, it should be taken into account that the optimal number of frames per second is 30 . By way of simple arithmetic calculations, we get the final number of frames equal to 9,000. Computation of each frame by an up-to-date computer takes some 30-33 seconds. Therefore, generation of a 9,000-frame video requires some 75 hours.

Visualization of the construction process of an industrial building should be performed in reversed order. It means that the animated building should be dismantled, rather than constructed. This is a correct 
approach to visualization of a construction process. It makes it possible to avoid piling-up of objects and, importantly, to reflect the construction process properly.

A factor of no small significance that should be borne in mind is the computing capacity of the computer. For the purpose of saving computer resources in computations, a camera is created in the $3 \mathrm{D}$ model that will limit the computation of the final image (Figure 2). Thus rendering of invisible sections of the created model can be avoided, and the whole of the computer's useful capacity can be involved in the computation of the required image.

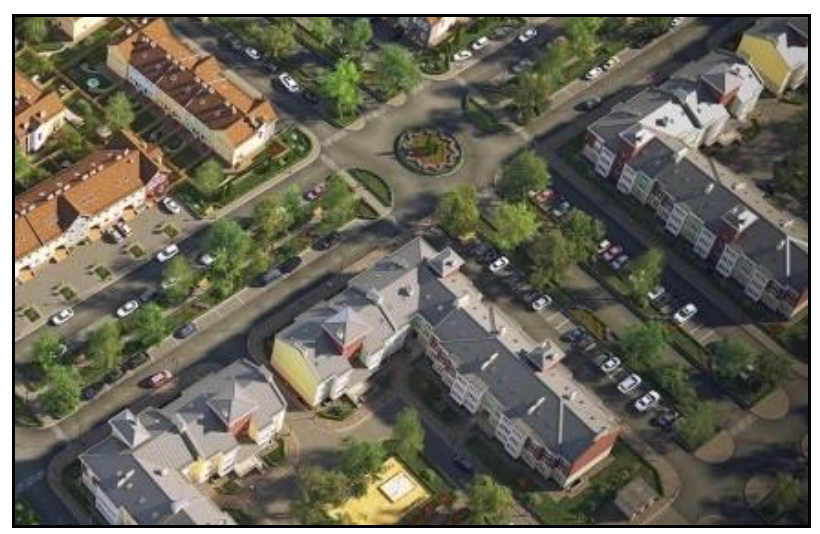

Fig. 2. Master plan, creating in 3 ds max.

The camera is also an object of the 3D model and has its own space coordinates. Having set the coordinates of the camera's frame-by-frame motion, we get an animation that depicts the existing situation. Such animations are generally used at the beginning and at the end of a video as a visual demonstration of the difference between the opening and ending frames.

There is no single construction master plan drawn up without layout and computation of the utilized construction machines. It is also required in animation, as the construction process of a building cannot be realistically represented if construction machines are not taken into account.

An important stage in the animation process is frameby-frame arrangement of objects of interaction with the building's model. An interval of appearance of each object and each part of the model should be specified for exact time. For example, the first object is a roller that rolls a road leading to the building. It is assigned a path of motion, and disappearance of the section of the road associated with the roller is adjusted in the ending frame. Thus, following the processing and inversion of visual imagery of the video file, an effect of laying an access road to the industrial building is achieved.

This technology is among the first steps in the improvement of the designing process for construction master plans.

One of the current trends in computer graphics development is photorealistic computer-modelled imaging.

Expansion of computing capacities offers solutions to progressively challenging tasks of 3D scene visualization (number of objects, sources of illumination, image resolution); however, it is outpaced by increasing complexity of these tasks.

The most widely used 3D modeling technique now is partial rearrangement of basic vector graphics. Its idea is as follows: all 3D models (of which, in the final account, the virtual world is composed) are represented as a certain number of intersecting plates. Excessive parts of the plates are cut off. The resulting products are 2D polygons placed in a $3 \mathrm{D}$ coordinate system.

One of the main trends in the development of computer-aided graphics is physically accurate modeling of light dissemination in various environments.

According to the outcome indicator, it has been ascertained that the software environment for virtual reality implementation in the construction master plan of the project is Autodesk 3ds Max.

This 3D modeling software package that has been developing since as early as the 1990s and has already reached a certain level of perfection. This is confirmed by its current popularity in the blossoming industries of movie-making, TV and computer games. Strikingly realistic 3D special effects on TV screens, stupendously lifelike virtual reality of 3D computer worlds, as well as numerous high-quality architectural and design projects implemented with the use of $3 \mathrm{~d}$ Max have become ingrained and occupied a prominent place in our everyday lives $[2,3,4]$.

It is worth noting that usually whole general construction plan is divided in parts and over every part is operated by a single engineer, designer or architect. This is significantly simplifying the work of specialists and increasing the whole stability of the software - it doesn't end the session prematurely, if the user doesn't want that to happen.

General construction plan is divided by individual files, for instance, in one single file a specialist draws buildings, in another - mechanisms and in the third one - roads and vegetation. All these files are attached to the general file, in which the separated elements of master plan are collected into one like puzzles. While specialists are working, not only their respective files are being updated but also the general file is updated with all the changes in real time.

Generation of a 3D model of a construction site. Modeling usually accounts for $50 \%$ to $80 \%$ of the whole work process. It is important to realize that the time consumed by modeling depends primarily on complexity of the situation on the site, and the building or the premises, rather than their dimensions. Model mapping time depends on complexity and diversity of the materials. Mapping a one-story industrial building is much easier and faster than a small cottage with various kinds of stone and facing bricks.

Visualization implies adjustment of lighting, environment and materials. At this stage, the computer model gets a photorealistic and presentable appearance, and the image quality is determined. This stage can account for $10 \%$ to $50 \%$ of the whole work process. The visualization quality depends directly on the experience and skills of the designer.

Working with light in $3 \mathrm{ds} \max$ is implemented through the render-software «V-ray» - it counts behavior 
of rays from sources of light on the surface of models and elements in the best way. Also such processing adds realism to finished 3D-models due to the generation of volumetric effect, the lights and darks of a picture and also reflections on the surfaces of different subjects.

The main cost elements of visualization are the scope and quality of work. A construction master plan developed in a usual format contains all required attributes. When the drawing is elevated, it is important to know how many and which aspects of the building should be reflected. This component has an impact on the time and quality of the reflected virtual reality. The color composition includes information on finishing materials and their coloring. It contains details of all basic elements from the pedestal to the roof and photos of material samples.

The resulting images may be of mixed quality. It is important to take into account the workstation resources. Working on an average power personal computer, entails the sacrifice of quality in animation or the decrease in number of viewpoints, which can be used to follow the course of animated models or to examine the whole general construction plan. If the process of visualization and subsequently that of animation are implemented on a so-called render farm, the outgoing quality of the image should not be downgraded. Using a standard computer means compromising on the animation quality, as the computer is simply unable to compute so many objects.

Computer-aided graphics make it possible to generate 3D virtual models of architectural projects of any degree of complexity and organization. 3D visualization is an important tool of attracting investment for a construction project, as the investor is shown not only unemotional calculations and engineering drawings, but also a vivid image of the future building. It is sometimes very difficult to describe a project concept using only traditional instruments such as drawings, sketches or photos of similar projects.

For a 3D object to be displayed on the screen online, it should be first presented in a dot form in a 3D coordinate system with each displayed dot having $\mathrm{x}, \mathrm{y}$ and $\mathrm{z}$ coordinates. Each object's dots that determine its location in space are stored in the system memory. In order to display an object on a 2D screen, it should be visualized.

Simple exterior scenes can be generated using "templates" available in $3 \mathrm{ds}$ Max starting from its $6^{\text {th }}$ version. Since the whole model is designed for animating the process of construction of an industrial building, all elements of the building are generated separately and combined into groups.

Technological process visualization is a way of displaying information about the state of technological equipment and technological process parameters on a computer monitor or an operator panel in an industrial automatic control system that also provides for graphic means of technological process control. The display system is based on a mnemonic diagram of the technological process, a static image that shows in visually simple and user-friendly elements of equipment, possibly, materials being processed and products, as well as their interaction and processing procedure. The static mnemonic diagram is animated reflecting the actual condition of the equipment and raw materials.

\section{Building data acquisition for master plans}

In most cases the construction is proceeding in the built up area. In order to demonstrate already existing buildings on the general construction plan, we can use the different methods of getting information about urban development in a given region.

Building models can be systematically derived by a wide range of techniques for acquisition, classification, and analysis of urban data derived from, for example, laser scans, aerial photography, and cadaster information bases. The initial creation is a technically challenging and economically cost-intensive task.

For more detailed buildings semi-automatic techniques of photogrammetry [8] are used or they are constructed by $\mathrm{CAD}$ or $3 \mathrm{D}$ modeling tools. The resulting buildings are represented, in general, using formats of 3D computer graphics (e.g., VRML or 3DS from Studio Max) because these models are supposed to be finalized by $3 \mathrm{D}$ graphics design applications, extending models by indoor and room features. Most notably buildings represented this way do not preserve semantics of building parts (e.g., classification of geometry parts in terms of architecture such as doors, windows, roofs, walls, staircases, etc.) unless special conventions are adopted.

Independently, procedural techniques for creating virtual 3D city models have emerged in the scope of computer graphics, intended for research, simulation, and educational purposes. In particular, specific markets such as the movie and game industry have a high demand for a cost and time-efficient creation of realistic, complex urban environments. Their representation, however, is tightly linked to and optimized for a specific visualization system. [5]

Parish and Müller [6] present a system that creates a complete 3D city model using a small set of statistical and geographical input data. The system provides tools for generating roads, allotments, buildings, and procedural textures. Wonka et al. introduce a concept for instant architectural building models. In their approach, building designs are derived using parametric set grammars, an attribute matching system, and a separate control grammar to derive buildings having a large variety of different styles and design ideas. In conclusion, procedural techniques, which are not concentrating on real-world geodata, do not need to address the heterogeneous building models and longterm maintenance. [5]

\section{City model representations}

Independent of the way of creation, virtual 3D city models can be exported as 3D scenes in standard 3D scene formats (e.g., VRML, X3D, or 3DS). While scene description languages and scene graph systems offer a broad repertoire of generic graphics functionality, they 
do not provide specialized means for 3D geodata-based objects. Consequently, it is generally difficult to represent, to preserve, and to take advantage of object semantics.

The CityGML initiative (Kolbe et al. [7]) addresses the need for a domain-specific, semantics-preserving description of virtual 3D city models based on XML. As Altmaier and Kolbe introduce, CityGML supports four different level-of-details:

- Block models derived by extruding ground plans to an average (measured or estimated) height (LOD-1);

- Block models including roof geometry and differentiated heights within a single building (LOD-2);

- Detailed building models with (LOD-3), and

- Architectural building models including indoor and room features such as doors, staircases (LOD-4).

The CityGML schema expresses principal quality levels as found in buildings delivered from today's acquisition techniques. To enhance this schema, we propose to add features that allow us to express building models between these discrete levels and facilitate the systematic refinement of a building model from one quality level to the next.

An integral solution for a continuous modeling across these quality levels would enhance the expressivity, address practically important intermediate quality levels, and enable developing efficient cross-LOD rendering and interaction techniques. For example, in many applications users want to transform an LOD-2 building into an LOD-4 building during a refinement process.

Conceptually, there is also no sharp distinction between LOD-2 and LOD-3 buildings: Even for LOD-2 buildings, it can be necessary to add significant geometric details such as an entrance hall if these details are perceptually important - the corresponding quality level could be considered to be between 2 and 3 . Similarly, in modern architecture the distinction between indoor and outdoor is softened, e.g., if large parts of a facade are made of glass, a minimal indoor model including floors and main walls would be required for a detailed building model - the corresponding quality level would be between 3 and 4 .

The concept of CLOQ buildings extends the CityGML modeling schema adopting the semantic schema but introducing an independent schema for modeling geometry and appearance.

\section{Conclusion}

All-round introduction of 3D graphics doesn't make designing of construction documents easier but makes it more expressive. Companies, which are using systems of $3 \mathrm{D}$ visualization, increase their competitive ability in the construction field. The use of 3D modeling systems is not only decreasing the number of blueprints but also allows us to show photorealistic view of the upcoming project, thus increasing the interest of investors and consumers in project realization.
Despite visible advantages of 3D visualization for general construction plan, let's not forget, that this feature requires a lot more investment than usual 2D blueprints: additional spending for powerful equipment and software is required. However, monetary investment does not ensure the obtainment of a perfect, available for free interaction model, because of limited capabilities of consumers and investors computers.

While modern computing equipment and screens boast rich graphic capabilities, the latter, if used extensively, may well contradict ergonomic requirements. Thus, a rich color palette cannot be used for displaying the state of a system. Information should be displayed in simple and unambiguously perceived colors (red, green, yellow, white, black, gray). Excessively small fonts should not be used in inscriptions either. Overuse of dynamic pictures (for example, animated images) distracts and overstrains the operator.

Photorealistic images of objects also hamper perception. For technological process control purposes, the mnemonic diagram includes graphic interface elements that are widely used in modern software packages, such as input-output windows, buttons, and sliders.

Image elements may also use events (for example, mouse clicks). A dynamic mnemonic diagram can be supplemented with special windows or above-diagram windows, which reflect time dependence of the technological process parameters and also text messages about the state of the system and actions of the operator. Modern design tools for operator control systems like SCAD typically contain built-in editors that make it possible to accomplish all visualization tasks. [5]

\section{References}

1. E.B. Kolesnikova, S.A. Sinenko, Virtual reality technology in displaying a construction master plan in construction operations, Industrial and Civil Engineering, No.11, p. 60 (2012)

2. I.M. Lebedeva, S.A. Sinenko, Algorithm for design solution visualization software in autocad ,Technology and Organization of Construction Operations, No. 1(1), p .43. (2012)

3. A.V. Ginzburg, A.A. Volkov,N.A. Gariaev, S.A. Sinenko, Design automation systems in construction operations: a learning guide for higher educational establishments - edited by Professor A.V. Ginzburg; Ministry of Education and Science of the Russian Federation, Moscow State University of Civil Engineering, Moscow (2014)

4. I.L. Abramov, T.Y. Poznakhirko, A. Sergeev, The analysis of the functionality of modern systems, methods and scheduling tools, MATEC Web of Conferences 86, 04063 (2016)

5. J. Döllner, H. Buchholz, Continuous Level-of-Detail Modeling of Buildings in 3D City Models, GIS '05 Proceedings of the 13th annual ACM international workshop on Geographic information systems, p. 173-181 (2005) 
6. Y. I. H. Parish, P. Müller, Procedural Modeling of Cities, Computer Graphics, p. 301-308. (Proceedings of ACM SIGGRAPH (2001)

7. T. H. Kolbe, G. Gröger, and L. Plümer, City GML Interoperable Access to $3 D$ City Models, First International Symposium on Geo-Information for Disaster Management GI4DM, (2005)

8. N. Haala, and C. Brenner, Laser Data for Virtual Landscape Generation (1999) 